\title{
Article
}

\section{What impact did a Paediatric Early Warning system have on emergency admissions to the paediatric intensive care unit? An observational cohort study}

Sefton, G., McGrath, C., Tume, Lyvonne Nicole, Lane, S., Lisboa, P.J.G. and Carrol, E.D.

Available at http://clok.uclan.ac.uk/11240/

Sefton, G., McGrath, C., Tume, Lyvonne Nicole ORCID: 0000-0002-2547-8209, Lane, S., Lisboa, P.J.G. and Carrol, E.D. (2014) What impact did a Paediatric Early Warning system have on emergency admissions to the paediatric intensive care unit? An observational cohort study. Intensive and Critical Care Nursing . ISSN 09643397

It is advisable to refer to the publisher's version if you intend to cite from the work. http://dx.doi.org/10.1016/j.iccn.2014.01.001

For more information about UCLan's research in this area go to http://www.uclan.ac.uk/researchgroups/ and search for <name of research Group>.

For information about Research generally at UCLan please go to http://www.uclan.ac.uk/research/

All outputs in CLoK are protected by Intellectual Property Rights law, including Copyright law. Copyright, IPR and Moral Rights for the works on this site are retained by the individual authors and/or other copyright owners. Terms and conditions for use of this material are defined in the policies page. 


\title{
What impact did a Paediatric Early Warning system have on emergency admissions to the paediatric intensive care unit? An observational cohort study
}

\author{
G. Sefton ${ }^{a}, *$, C. McGrath ${ }^{a}$, L. Tume ${ }^{a}$, S. Lane ${ }^{b}$, P.J.G. Lisboa ${ }^{c}$, \\ E.D. Carrol $^{\mathrm{b}}$ \\ a PICU, Alder Hey Children's NHS Foundation Trust, Eaton Road, Liverpool L12 2AP, United Kingdom \\ b University of Liverpool, Brownlow Hill, Liverpool L69 7ZX, United Kingdom \\ c John Moores University, Byrom Street, Liverpool L3 3AF, United Kingdom
}

Accepted 6 January 2014

\author{
KEYWORDS \\ Child; \\ Deterioration; \\ Emergency; \\ Paediatric; \\ Paediatric Early \\ Warning; \\ Pediatric; \\ PEW; \\ PICU; \\ Unplanned admission \\ to PICU
}

\begin{abstract}
Summary The ideology underpinning Paediatric Early Warning systems (PEWs) is that earlier recognition of deteriorating in-patients would improve clinical outcomes.

Objective: To explore how the introduction of PEWs at a tertiary children's hospital affects emergency admissions to the Paediatric Intensive Care Unit (PICU) and the impact on service delivery. To compare 'in-house' emergency admissions to PICU with 'external' admissions transferred from District General Hospitals (without PEWs).

Method: A before-and-after observational study August 2005-July 2006 (pre), August 2006-July 2007 (post) implementation of PEWs at the tertiary children's hospital.

Results: The median Paediatric Index of Mortality (PIM2) reduced; 0.44 vs $0.60(p<0.001)$. Fewer admissions required invasive ventilation $62.7 \%$ vs $75.2 \%(p=0.015)$ for a shorter median duration; four to two days. The median length of PICU stay reduced; five to three days $(p=0.002)$. There was a non-significant reduction in mortality $(p=0.47)$. There was no comparable improvement in outcome seen in external emergency admissions to PICU. A 39\% reduction in emergency admission total beds days reduced cancellation of major elective surgical cases and refusal of external PICU referrals.

Conclusions: Following introduction of PEWs at a tertiary children's hospital PIM2 was reduced, patients required less PICU interventions and had a shorter length of stay. PICU service delivery improved.
\end{abstract}

(c) 2014 Elsevier Ltd. All rights reserved.

* Corresponding author. Tel.: +44 1512824792.

E-mail address: gerri.sefton@alderhey.nhs.uk (G. Sefton).

0964-3397/\$ - see front matter @ 2014 Elsevier Ltd. All rights reserved.

http://dx.doi.org/10.1016/j.iccn.2014.01.001 


\section{Implications for Clinical Practice}

Following the introduction of a Paediatric Early Warning system at a tertiary children's hospital, 'in-house' emergency admissions to PICU:

- Had a lower Prognostic Indicator of Mortality score (PIM2) at first contact with the PICU team: 0.44 vs 0.60 ( $p<0.001$ ).

- Had a lower requirement for intubation and mechanical ventilation $62.7 \%$ vs $75.2 \%(p=0.015)$ for a shorter median duration; four to two days.

- Had a reduced median length of PICU stay five to three days $(p=0.002)$.

There was a $39 \%$ reduction of the total bed days used for emergency admissions to PICU which improved PICU service delivery

- Cancellation of major elective surgical cases reduced by $90 \%$.

- Refused regional PICU referrals reduced a $79 \%$.

\section{Introduction}

The reported incidence of cardiopulmonary arrest in hospitalised children is low: 0.2-2.5/1000 (Berg et al., 2008). However, mortality (52\%) and morbidity remain high despite advances in resuscitation training, technology and treatment (Tibballs et al., 2005). The devastating physical consequences of deterioration leading to cardiopulmonary arrest are well documented (Meert et al., 2009), as are the psychological effects that emergency admission to the Paediatric Intensive Care Unit (PICU) has on the family (Balluffi et al., 2004). There are significant additional financial costs to the National Health Service of 'failing to rescue' deteriorating children in hospital (Duncan and Frew, 2009). Therefore, there is a pressing social and financial need to improve the early identification and treatment of deterioration in hospitalised children.

Research in adults demonstrated that cardiopulmonary arrest or emergency admissions to Intensive Care were often preceded by a period of physiological instability which, once recognised, provided an opportunity for the healthcare team to intervene to improve outcome (Hodgetts et al., 2002; Kause et al., 2004). A similar window of opportunity may exist for hospitalised children (Haines, 2005; Tasker, 2005; Tume, 2004, 2006).

Paediatric Early Warning systems (PEWs) have been identified as a mechanism to improve safety for hospitalised children (CEMACH, 2008). To date, published studies evaluating PEWs have focused on the performance of individual tools in single centres and their impact on the incidence of respiratory or cardiopulmonary arrest. However, this does not capture other sick children admitted to the PICU as emergencies following acute deterioration or the impact that PEWs has on PICU service delivery.

\section{Study objectives}

The study objectives were

1. To understand how the introduction of a Paediatric Early Warning system at a tertiary children's hospital affects emergency admissions to the PICU.
2. To compare the 'in-house' cohort of emergency admissions to PICU with a comparable group; emergency admissions transferred to PICU from wards at District General Hospitals (without PEWs in place).

3. To explore the impact that a PEW system had on PICU service delivery.

\section{Methods}

\section{Setting}

The setting was a tertiary children's hospital in the Northwest of England, with 337 in-patient beds, handling 37,000 annual admissions (excluding day-cases). Tertiary specialties include cardiology/cardiac surgery, neurology/neurosurgery, renal, oncology, burns/plastics and neonatal surgery. The hospital has a 24 hour emergency department, a 22 bed PICU and two separate High Dependency Units (total beds 21). The PICU admits 1100 patients annually. Half of those admissions are elective, following major surgery including cardiac surgery or neurosurgery. The remaining admissions are unplanned; from the emergency department, theatre, wards within the hospital or urgent transfers from any of 28 District General Hospitals (DGHs) within the region.

\section{Intervention-implementation of PEWs at tertiary children's hospital}

In 2006, a study based at this hospital (Tume, 2006) showed that emergency admissions to PICU or High Dependency were often preceded by a period of documented abnormal observations which were either not recognised or not responded to quickly enough to halt deterioration. A decision was made to implement locally adapted based on the Bristol PEW; (Haines, 2005) (Fig. 1) to improve the early recognition of deterioration so that the healthcare team had a greater opportunity to intervene to improve outcome.

A project group [Resuscitation Training Officer, General Paediatrician, Critical Care Physiotherapist, Advanced Nurse Practitioner PICU, Intensivist, PICU and High Dependency Lecturer Practitioners, PEWs project nurse, senior ward 
PAEDIATRIC EARLY WARNING TOOL

Each time the vital signs are taken, they must be cross referenced against the PEW criteria.

The tool is considered to be triggered if any one or more of the parameters are breached. Follow actions below

\begin{tabular}{|ll|}
\hline A & ACUTE AIRWAY OBSTRUCTION (seek prompt assistance) \\
1) Child requires nebulised Adrenaline $\underline{\text { OR no improvement after nebulised Adrenaline }}$ \\
2) & Clinically tiring or impending complete airway obstruction \\
\hline
\end{tabular}

$\begin{array}{ll}\text { B } & \text { BREATHING } \\ \text { 1) } & \mathrm{SaO}_{2} \leq \mathbf{9 2} \% \text { in any amount of oxygen } \\ \text { 2) } & \mathrm{SaO}_{2} \leq 75 \% \text { in any amount of oxygen (cyanotic heart disease) }\end{array}$

2) $\mathrm{SaO}_{2} \leq 75 \%$ in any amount of oxygen (cyanotic heart disease)

3) Persistent tachypnoea

(RR $\geq \mathbf{7 0}$ under 6 months; $\geq \mathbf{6 0} 6-12$ months; $\geq \mathbf{4 0} 1-5 \mathrm{yrs} ; \mathbf{2} \mathbf{2 5}$ over $5 \mathrm{yrs}$ )

4) Apnoeas + /- bradycardia (HR $\leq 95$ in children under 5 yrs)

5) Marked increased effort of breathing (3+ on table front sheet)

6) Respiratory depression $\mathbf{R R}<\mathbf{2 0} 0-3$ months, $\leq$ half lower value for resps for age (table front sheet)

\begin{tabular}{|ll|}
\hline C & CIRCULATION \\
1) & Persistent tachycardia following one bolus of $10 \mathrm{mls} / \mathrm{kg}$ fluid \\
& (HR $\geq \mathbf{1 5 0}$ under 5 yrs; $H R \geq \mathbf{1 2 0} 5-12$ years; $H R \geq \mathbf{1 0 0}$ over 12 yrs) \\
2) & Poor perfusion; prolonged capillary refill ( $\geq \mathbf{3}$ secs); $+/$ - low $B P$, large central/peripheral temp gradient \\
\hline
\end{tabular}

\begin{tabular}{|ll}
\hline D & DISABILITY \\
1) & GCS $\leq \mathbf{1 1}$ or falling. Children scored by AVPU; responding only to pain $\mathrm{OR}$ unresponsive \\
2) & Fitting; unexpected $\mathrm{OR}$ not responding to prescribed anticonvulsants \\
\hline
\end{tabular}

\begin{tabular}{|ll|}
\hline E & OTHERS \\
1) & Hyperkalaemia $-\mathbf{K}^{+} \geq \mathbf{6 . 0}$ mmol/litre \\
2) & Any child with $\mathrm{pH}<7.2$ whatever the cause \\
3) & Any child with unresolved pain on current analgesic therapy \\
4) & Any child whose condition is worrying - but not triggering on above parameters \\
\hline
\end{tabular}

\begin{tabular}{|c|c|}
\hline $\begin{array}{l}\text { Action to be taken if the PEW } \\
\text { tool is triggered } \\
\text { This tool does not replace } \\
\text { clinical judgement. If the child } \\
\text { is deteriorating rapidly, or peri- } \\
\text { arrest put out an arrest call } \\
\text { immediately - Dial } 2222 \\
\text { Medical review expected within } 30 \\
\text { minutes, within } 10 \text { minutes if it is an } \\
\text { airway trigger } \\
\text { Otherwise } \\
\text { - Alert the nurse in charge on } \\
\text { the ward. } \\
\text { - Increase the } \\
\text { frequency of observations. } \\
\text { - Notify Patient's own medical } \\
\text { team. If the team cannot be } \\
\text { contacted or cannot attend, } \\
\text { contact the on -take team to } \\
\text { review \& stabilise the child } \\
\text { Out of hours contact the on } \\
\text { call team and inform the } \\
\text { Night Matron } \\
\text { Complete PEW tool } \\
\text { assessment on meditech } \\
\text { under nursing assessment } \\
\text { Children triggering PEW } \\
\text { must be discussed with } \\
\text { the registrar }\end{array}$ & $\begin{array}{l}\text { If the patient already transgresses the above criteria, the } \\
\text { medical team responsible for the childs care can set alternative } \\
\text { acceptable Paediatric Early Warning parameters. The PEW tool is } \\
\text { triggered when the alternative parameters are breached. }\end{array}$ \\
\hline
\end{tabular}

Figure 1 Modified Bristol Paediatric Early Warning tool. Adapted from Haines (2005).

nurses] prepared the processes to underpin the organisational implementation of PEWs. A clinical policy was ratified which outlined the standard required for monitoring and observation of in-patients.

The modified Bristol PEW (Haines, 2005) tool was incorporated within patient observation charts. The chart format followed an 'Advanced Paediatric Life Support' (APLS, 2005) structured approach, first assessing Airway, then Breathing, Circulation and Disability $(A, B, C, D)$. Thresholds for concern for respiratory rate, heart rate and blood pressure were based on APLS guidelines. Focused PEWs training was implemented for all frontline clinical hospital staff (standard for 
taking observations, documentation, recognising abnormal observations and escalation of concern). The new charts were introduced in all in-patient areas in August 2006.

The PEWs was considered to be activated if one or more of the thresholds for abnormal observations were breached. The hospital had a cardiac arrest team to respond to respiratory or cardiac arrests, but did not have a Medical Emergency Team or a Rapid Response Team. The Resuscitation committee agreed the following clinical response to patients activating the PEWs (Table 1).

Table 1 Locally agreed clinical response system for patients triggering PEWs.

- Existing medical/surgical teams will respond to their own patients if they trigger PEWs.

- The on-call medical/surgical team will respond to patients triggering PEWs outside of day-time working hours Monday-Friday, weekends, bank holidays OR if the child's own medical team cannot be contacted.

- Target response times will be 'within 10 minutes' for an airway trigger and 'within 30 minutes' for all other triggers.

- Alternative trigger criteria can be set by the medical team responsible for the patient.

- In specific cases e.g. $\mathrm{SpO}_{2}>75 \%$ for severe cyanotic heart defects.

- PEWs will not replace clinical judgement i.e. if a child is deteriorating rapidly or peri-arrest, the usual procedure of calling the arrest team will be followed.

- The PICU team will continue to review deteriorating patients as requested.

\section{Study design}

This is an observational cohort study, conducted at a large tertiary children's hospital in the United Kingdom. A summary of the study design is presented in Table 2

Patient-specific information and source data is routinely collected prospectively for all PICU admissions for the Paediatric Intensive Care Audit Network dataset (PICANet) (Table 2). The data is rigorously checked by random case quality control each month and is considered to be both accurate and reliable. Formal permission was not required to use this centre's own PICANet data. The study was registered as clinical audit/service evaluation and followed the NHS Trust guidance for audit and governance.

Over the same time period, the hospital Informatics team collected data about the unmet demands for PICU beds. This included cancellation of major elective surgery and refusal of external PICU referrals when no PICU beds were available. This gave valuable information on PICU service delivery (Table 2).

\section{Paediatric Index of Mortality (PIM2)}

Paediatric Index of Mortality (PIM2) is calculated for all PICU admissions at first contact with the PICU team. It is a measure of the likelihood of dying during the PICU admission (Slater et al., 2003). It is useful to compare different systems of intensive care and for describing severity of illness for groups of patients in research studies. It is not intended for use as an assessment of individual patient severity of illness. Therefore, in this study the requirement for invasive ventilation and inotropes were used as surrogate markers for severity of illness.

\section{Results}

A total of 958 unplanned PICU admissions over two years were reviewed, for one year before PEWs introduction and one year afterwards.

\section{The 'In-house' cohort}

The 'in-house' cohort (Table 3) was demographically similar during both time periods. $60 \%$ of admissions were less than one year of age, with marginally more males.

Having PEWs in place did not reduce the incidence of emergency admissions to PICU. However, the median PIM2 score dropped to 0.44 from $0.60(p<0.001)$, indicating that the likelihood of dying during the PICU admission had reduced significantly. This suggests that this patient cohort had been identified at an earlier point in their illness.

Fewer admissions required invasive ventilation; $62 \%$ vs $75 \%(p=0.015)$ for a shorter median duration, dropping from four to two days.

The proportion of admissions requiring inotropic support reduced from $31.8 \%$ to $24 \%$, which was not statistically significant $(p=0.12)$. The median duration of inotropic support was unchanged. The median length of stay on PICU reduced from five to three days $(p=0.002)$. There was a nonsignificant reduction in mortality [10.8 to $8.4 \%(p=0.47)]$, consistent with the reduction in PIM2 score.

\section{The 'External' cohort}

In the external cohort (Table 4) the numbers of emergency admissions transferred from DGHs to the tertiary PICU, were similar over both time periods. However, more infants, median age of three months, were transferred 2006/2007. The median PIM2 scores at first contact with the PICU team increased from 0.060 to 0.072 , suggesting an increased likelihood of dying during the PICU admission.

The requirement for intubation and ventilation, inotropic support and the median length of stay were similar in the before-and-after groups. There was a marginal improvement in mortality from $10.6 \%$ to $8.2 \%$, which was not statistically significant $(p=0.30)$.

\section{The impact that PEWs had on PICU service delivery}

PICU service delivery is affected by the number of patients admitted as emergencies, their severity of illness and their length of stay. The effect of mismatched PICU bed availability and demand include cancellation of major elective surgical cases at short notice or refusal of external referrals for PICU. This has clinical and cost implications. If there are no available PICU beds in the region, the critically ill 
Table 2 Methodology summary.

\begin{tabular}{|c|c|}
\hline & Description \\
\hline Study design & Observational cohort study \\
\hline \multicolumn{2}{|l|}{ Population } \\
\hline - 'In-house' cohort & $\begin{array}{l}\text { All emergency admissions admitted to the tertiary PICU from wards } \\
\text { within the same hospital. }\end{array}$ \\
\hline - 'External' cohort & $\begin{array}{l}\text { All emergency admissions admitted to the tertiary PICU transferred } \\
\text { from wards within the District General Hospitals (DGH). }\end{array}$ \\
\hline Intervention & $\begin{array}{l}\text { Introduction of the modified Bristol Paediatric Early Warning (PEWs) } \\
\text { (Haines, 2005) tool at the tertiary children's hospital in August } 2006 \\
\text { No PEWs was in use at the DGHs } 2005 \text { to } 2007 . \text { Routine practice } \\
\text { continued. }\end{array}$ \\
\hline Comparison & $\begin{array}{l}\text { PICU outcomes for the' in-house' and 'external' cohort of emergency } \\
\text { admissions to the tertiary PICU over a two year period } \\
\text { - One year pre PEW introduction August 2005-July } 2006 \\
\text { - One year post PEW introduction August 2006-July } 2007\end{array}$ \\
\hline \multicolumn{2}{|l|}{ Data collection and outcomes measured } \\
\hline $\begin{array}{l}\text { - Paediatric Intensive Care Audit Network } \\
\text { dataset (data from own centre) }\end{array}$ & $\begin{array}{l}\text { Age (months), gender, Diagnostic group, Paediatric Index of Mortality } \\
\text { (PIM2) at first contact with the PICU team, PICU interventions } \\
\text { required, length of PICU stay (days) and PICU mortality. }\end{array}$ \\
\hline - Hospital Informatics dataset & $\begin{array}{l}\text { The number of refused regional referrals for PICU, due to the } \\
\text { non-availability of a PICU bed. } \\
\text { The number of cancelled major elective surgical cases, due to the } \\
\text { non-availability of a PICU bed. }\end{array}$ \\
\hline Statistical analysis & $\begin{array}{l}\text { PICANet data from an ACCESS (Microsoft) database was exported to } \\
\text { SPSS } 18.0 \\
\text { - checked for accuracy } \\
\text { - descriptive analysis: percentages, median and interquartile range } \\
\text { (IQR) } \\
\text { - inferential data analysis: } \\
\text { Categorical data was analysed using the chi-squared test. } \\
\text { Continuous data was not normally distributed so was analysed using } \\
\text { the Mann Whitney } U \text { test. } \\
\text { - } p \text { value }<0.05 \text { was considered significant. }\end{array}$ \\
\hline
\end{tabular}

child would have to be transferred out of region, with the added clinical risks of a longer transfer time to another PICU, increased disruption for the child's family and a higher cost to the local health authority.

There are also penalties for late cancellation of elective surgery. In the UK, Government targets dictate that cancelled elective surgery on the day of surgery must be rescheduled within four weeks (NHSLA, 2012-2013), which places increased pressure on theatre waiting lists. Therefore there is an on-going impetus to optimise the PICU bed utilisation/productivity.

In this study, the shorter length of PICU stay for the 'in-house' emergency admissions translated into improved productivity. The cumulative effect of shorter length of stay meant there was a $39 \%$ overall reduction in the total number of bed days used for emergency PICU admissions. Cancellation of major elective surgical cases was reduced by $90 \%$ (Table 5). There was also a 79\% reduction in the number of refused regional PICU referrals.

\section{Discussion}

In the United Kingdom, the National Patient Safety Association (2009), Confidential Enquiry into Maternal and
Child Health deaths (CEMACH, 2008) and NHS litigation authority (NHSLA, 2012-2013) have advocated for the widespread use of PEWs, to assist staff in the early identification of children who may be deteriorating. This advice is made based on consensus opinion and as yet the evidence to support this is limited.

In practice, it is recognised that evaluating a complex intervention like PEWs is difficult (Craig et al., 2008). In paediatrics, there is currently no consensus on which specific observations should be used to predict deterioration in sick children (Chapman et al., 2010). A large number of PEWs (many un-validated) are in use within the United Kingdom (Roland et al., 2013). There is considerable heterogeneity amongst the alert criteria used, with variation of age specific reference ranges used to signify 'abnormal vital signs', making it currently impossible to compare studies. Additionally, the success of PEWs depends not only on the tool's predictive ability to appropriately signal deterioration but also on having adequate organisational resource to allow close observation of sick children, recognise those 'at risk', escalate the concern and generate a prompt and appropriate medical response.

Published studies in paediatrics have described the implementation of PEWs in conjunction with a specialised 
Table 3 Emergency admissions to PICU: 'In-house' cohort.

\begin{tabular}{|c|c|c|c|}
\hline & Pre PEWs & Post PEWs & $p$ value \\
\hline Emergency admissions (n)/total PICU admissions (n) [\%] & $157 / 1087[14.4 \%]$ & $166 / 1039$ [16\%] & \\
\hline Median age months $(\mathrm{IQR})^{\mathrm{c}}$ & $7(38)$ & $8(34)$ & \\
\hline $0-1$ years $(n)$ (\% emergency admissions) & $97(61.8)$ & $99(59.6)$ & \\
\hline $1-2$ years & $13(8.3)$ & $14(8.4)$ & \\
\hline $2-7$ years & $18(11.5)$ & $25(15.1)$ & \\
\hline $7-13$ years & $19(12.1)$ & $14(8.4)$ & \\
\hline Over 13 years & $10(6.4)$ & $14(8.4)$ & \\
\hline Male & $57.3 \%$ & $57.2 \%$ & \\
\hline \multicolumn{4}{|l|}{ Diagnostic groups $\boldsymbol{n}$ (\% emergency admissions) } \\
\hline Respiratory & $95(60.5)$ & $102(61.4)$ & \\
\hline Cardiac & $27(17.2)$ & $17(10.2)$ & \\
\hline Neurology & $12(7.6)$ & $16(9.6)$ & \\
\hline Trauma & 0 & $3(1.8)$ & \\
\hline Sepsis & $15(9.6)$ & $11(6.6)$ & \\
\hline Gen surgery & 0 & $9(5.4)$ & \\
\hline Others (ENT, renal, gastro, oncology) & $8(5.1)$ & $8(4.8)$ & \\
\hline \multicolumn{4}{|l|}{ Outcomes } \\
\hline PIM2 median (IQR) & $0.060(0.078)$ & $0.044(0.049)$ & $p<0.0011^{a}$ \\
\hline Invasive ventilation $(n)(\%)$ & $118(75.2)$ & $104(62.7)$ & $p=0.0152^{b}$ \\
\hline Median length of ventilation days (IQR) & $4(11)$ & $2(5)$ & \\
\hline Inotropes $(n)(\%)$ & $50(31.8)$ & $40(24.1)$ & $p=0.122^{b}$ \\
\hline Median length of inotropes days (IQR) & $0(2)$ & $0(0)$ & \\
\hline PICU LOS days; median (IQR) & $5(9)$ & $3(5)$ & $p=0.0021^{a}$ \\
\hline PICU mortality $(n)(\%)$ & $17(10.8)$ & $14(8.4)$ & $p=0.472^{b}$ \\
\hline
\end{tabular}

Medical Emergency Team or Rapid Response Team, which makes it difficult to quantify the impact of either intervention in isolation. These specialised teams, although slightly different in their make-up, are experienced in recognising and responding proactively to deteriorating children. For ease, the umbrella term Medical Emergency Team will be used here to describe this response.

Tibballs and Kinney (2009) compared data from a tertiary paediatric hospital in Australia for 41 months prior to the introduction of a Medical Emergency Team with 48 months post intervention. They found a $55 \%$ reduction in preventable cardiac arrests (in patients who would have fulfilled their activation criteria) and a $34 \%$ reduction in mortality. Sharek et al. (2007) compared data from a quaternary paediatric hospital in USA for 56 months prior to the introduction of a Medical Emergency Team with 19 months post intervention. They found a $72 \%$ reduction in cardiac arrests and an $18 \%$ reduction in mortality. Both studies used historical controls from their own centre, a methodology which has since been challenged (Joffe et al., 2011). Joffe's group highlighted the methodological limitations of using historical controls to demonstrate the effectiveness of an intervention. During the same time periods as those published studies, there was a significant reduction in mortality at their Canadian quaternary Children's hospital without the use of PEWs or a Medical Emergency Team. This raises the possibility that the improved mortality might be related to organisational factors or even that mortality naturally improved over time.

Our study showed a statistically significant reduction in the risk of dying during the PICU admission (PIM2) in emergency 'in-house' admissions from wards within the tertiary paediatric hospital, following introduction of a Paediatric Early Warning system. However, that did not translate to a statistically significant reduction in mortality. A larger population would need to be studied to demonstrate an effect.

In the external cohort of emergency admissions to PICU from the DGHs, it is unclear why the likelihood of dying during the PICU admission (PIM2) increased. Paediatric Early Warning systems were not in place at the DGHs at this time (personal communication), so recognition and treatment of deterioration was dependent on experienced nurses and medical staff attending to the patient. Interestingly, the average mortality for both in-house and external emergency admissions was $9.5 \%$. This is much higher than the overall PICU mortality for this centre which runs at $3.5-4 \%$. Increased mortality of emergency admissions to PICU has also been described in adults (Goldhill and McNarry, 2004) and paediatrics (Odetola et al., 2007). 
Table 4 Emergency admissions to PICU: External cohort (from DGHs).

\begin{tabular}{|c|c|c|c|}
\hline & Aug 2005-July 2006 & Aug 2005-July 2006 & $p$ value \\
\hline Unplanned admissions ( $n$ )/total PICU admissions (n) [\%] & $315 / 1087$ [29\%] & $320 / 1039[30.8 \%]$ & \\
\hline Median age months $(\mathrm{IQR})^{\mathrm{c}}$ & $6(47)$ & $3(47)$ & \\
\hline $0-1$ years $n$ (\% emergency admissions) & $183(58.1)$ & $174(54.4)$ & \\
\hline $1-2$ years & $24(7.6)$ & $33(10.3)$ & \\
\hline $2-7$ years & $56(17.8)$ & $48(15)$ & \\
\hline $7-13$ years & $18(5.7)$ & $33(10.3)$ & \\
\hline Over 13 years & $34(10.8)$ & $32(10)$ & \\
\hline Male & $63 \%$ & $60.6 \%$ & \\
\hline \multicolumn{4}{|l|}{ Diagnostic groups $N(\%)$} \\
\hline Respiratory & $62(19.7)$ & $90(28.1)$ & \\
\hline Cardiac & $64(20.3)$ & $51(15.9)$ & \\
\hline Neurology & $37(11.7)$ & $53(16.6)$ & \\
\hline Trauma & 47 (14.9) & $30(9.4)$ & \\
\hline Sepsis & $40(12.7)$ & $42(13.1)$ & \\
\hline Gen surgery & $42(13.3)$ & $28(8.8)$ & \\
\hline Others (ENT, renal, gastro, oncology) & $23(7.3)$ & $26(8.1)$ & \\
\hline \multicolumn{4}{|l|}{ Outcomes } \\
\hline PIM2 median (IQR) & $0.060(0.075)$ & $0.072(0.078)$ & $p=0.131^{a}$ \\
\hline Invasive ventilation $(n)(\%)$ & $304(96.5)$ & $303(95.4)$ & $p=0.552^{b}$ \\
\hline Median length of ventilation days (IQR) & $3(5)$ & $3(3)$ & \\
\hline Inotrope $(n)(\%)$ & $92(29.2)$ & $99(31.2)$ & $p=0.582^{b}$ \\
\hline Median inotrope $(n)$ (IQR) & $0(2)$ & $0(2)$ & \\
\hline PICU LOS days; median (IQR) & $3(4)$ & $3(5)$ & $p=0.191^{a}$ \\
\hline PICU mortality $(n)(\%)$ & $31(10.6)$ & $26(8.2)$ & $p=0.192^{b}$ \\
\hline
\end{tabular}

The 'in-house' cohort also required less PICU interventions and had a shorter PICU length of stay, compared to the external cohort. This may be related to the close proximity to the PICU and the ease of referral and escalation of care. Almost $40 \%$ of the 'in-house' cohort did not require invasive ventilation. Increased access to HDU beds could have reduced the requirement for emergency admission to PICU.

The higher proportion of invasively ventilated patients in the 'external' cohort may be explained as a process bias. Invasive ventilation is the mechanism for safely transferring a critically ill child from their base hospital to the PICU.
If a patient was intubated and ventilated for safe transfer, it would be reasonable to expect that the patient would extubate promptly following safe arrival at the receiving PICU. However, in this study, the median duration of ventilation was three days, suggesting that few children required intubation and ventilation just for safe transfer.

To our knowledge, there are no previous published comparisons of the impact of PEWs on emergency admissions to the PICU. Balancing the requirement for elective PICU admissions against the unpredictable number of emergency admissions, with the fixed number of PICU beds, is

Table 5 Impact on PICU service delivery following the introduction of PEWs at a tertiary children's hospital.

\begin{tabular}{|c|c|c|}
\hline & $\begin{array}{l}\text { Aug 2005-July } \\
2006 \text { PRE-PEW }\end{array}$ & $\begin{array}{l}\text { Aug 2006-July } \\
2007 \text { POST-PEW }\end{array}$ \\
\hline $\begin{array}{l}\text { Cancelled major surgical theatre cases due to non-availability } \\
\text { of PICU bed }(n)\end{array}$ & 20 & 2 \\
\hline $\begin{array}{l}\text { Refusal of external referrals of sick children requiring PICU } \\
\text { care, due to non-availability of PICU bed }(n)\end{array}$ & 130 & 27 \\
\hline Total bed days used in-house emergency admissions ( $n$ ) (IQR) & $1569(411)$ & $994(253)$ \\
\hline Range bed days used for in-house emergency admit (IQR) & $1-163(2-11)$ & $1-55(1-6)$ \\
\hline
\end{tabular}


challenging. Invariably emergencies take priority over elective admissions, impacting on service delivery. National PICU audit data (PICANet, 2011) showed that emergency admissions to PICUs from wards within the same hospital account for over 2700 admissions in the United Kingdom per year, with 3200 additional emergency admissions transferred from DGHs wards. While there are limited possibilities for clinicians to impact on the number of sick children admitted to PICU from the emergency department, this study suggests there may be an opportunity to impact on the clinical course of paediatric in-patients who deteriorate in hospital, thereby reducing emergency admissions to PICU.

\section{Limitations}

This is a small before-and-after observational study undertaken at a single centre. We acknowledge the limitations of this study design, as described by Joffe et al. (2011) but have attempted to address these by using a comparison group. No attempt was made to test the performance of the individual PEW criteria during this study, or the local compliance with the PEW process, which may have impacted on its effectiveness. There may have been inaccuracies in the timing of the external referral to PICU and arrival of the patient at the tertiary PICU centre, so no judgement could be made on timelines of transfer from DGHs, which may impact on the outcomes of this group. Data on activation of the cardiac arrest team (Codes) were not used in this comparison, as there is no access to that data or denominator data from the DGHs.

No comment can be made about staffing levels, or ward management structure at the tertiary centre or DGHs, as this information was not collected. The staff at the tertiary centre received additional training on recognition of the deteriorating child and the process for escalation of concern. No data is available on the training given to staff at the DGHs. It is possible that these variables could impact on patient outcome (Diya et al., 2012).

\section{Conclusion}

Although there is limited evidence of effectiveness for using PEWs, conceptually it makes sense that if deteriorating children could be identified earlier, they would be easier to rescue. Indeed, there is no published evidence of harm from using PEWs. Despite its limitations, this study showed that the implementation of PEWs at a tertiary paediatric hospital reduced the likelihood of dying during the PICU admission, the requirement for PICU interventions and the PICU length of stay for 'in-house' emergency admissions. This reduced the total bed days utilised by emergency admissions at a large PICU in the United Kingdom, improving the availability of PICU beds.

In hospitals delivering in-patient paediatric healthcare, PEWs can be introduced at minimal cost to aid the early recognition of the deteriorating child. This may have relevance in resource limited healthcare settings worldwide. The perception that a formal specialised response team is required to implement PEWs may have hindered widespread uptake, due to financial constraints. Further work is required in a larger multi-centre study to determine if mortality and morbidity are reduced by the introduction of PEWs.

\section{Contributions}

Concept, study design G.S. and C.M. Data collection and analysis G.S., C.M. and S.L. Manuscript preparation G.S., L.T., S.L., P.L. and E.C.

\section{Conflict of interest}

None declared.

\section{Acknowledgement}

The authors would like to thank Elaine Scott for helping prepare the PICANet data for transfer to SPSS for further analysis.

This project to introduce a PEWs using existing resources to respond to deteriorating patients, won the BUPA patient safety award for 2007.

\section{References}

APLS. A structured approach to the seriously ill child. In: Samuels M, Wieteska S, editors. Advanced paediatric life support: the practical approach. 5th ed. UK: Advanced Life Support Group; 2005. p. 55 [chapter 7].

Balluffi A, Kassam-Adams N, Kazak A, Tucker M, Dominguez T, Helfaer M. Traumatic stress in parents of children admitted to the pediatric intensive care unit. Paediatric Critical Care Medicine 2004;5(November (6)):547-53.

Berg MD, Nadkarni VM, Zuercher MRA. In-hospital pediatric cardiac arrest. Pediatric Clinics of North America 2008;55:589-604.

CEMACH.Pearson GA, editor. Why children die: a pilot study 2006; 2008, http://www.cemach.org.uk/ [accessed 01.05.13].

Chapman SM, Grocott MPW, Franck LS. Systematic review of paediatric alert criteria for identifying hospitalised children at risk of critical deterioration. Intensive Care Medicine 2010;36(4):600-11.

Craig P, Dieppe P, Macintyre S, Michael S, Nazareth I, Pettigrew M. Developing and evaluating complex interventions - new guidance. Medical Research Council (MRC). Critical Care 2008;15(4):R184, http://dx.doi.org/ 10.1186/cc103370penAccess.

Diya L, Van den Heede K, Sermeus W, Lesaffre E. The relationship between in-hospital mortality, readmission into the intensive care nursing unit and/or operating theatre and nurse staffing levels. Journal of Advanced Nursing 2012;68(5):1073-81.

Duncan $\mathrm{H}$, Frew E. Short-term health system costs of paediatric inhospital acute life-threatening events including cardiac arrest. Resuscitation 2009;80(5):529-34.

Goldhill DR, McNarry AF. Physiological abnormalities in early warning scores are related to mortality in adult inpatients. British Journal of Anaesthesia 2004;92(6):882-4.

Haines C. Acutely ill children within ward areas - care provision and possible development strategies. Nursing in Critical Care 2005; 10:98-104.

Hodgetts TJ, Kenward G, Vlackonikolis I, Payneb S, Castle N, Crouch $\mathrm{R}$, et al. Incidence, location and reasons for avoidable in-hospital cardiac arrest in a district general hospital. Resuscitation 2002; 54:115-23.

Joffe AR, Anton NR, Burkholder SC. Reduction in hospital mortality over time in a hospital without a pediatric medical emergency 
team: limitations of before-and-after study designs. Archives of Pediatric and Adolescent Medicine 2011;165(5):419-23.

Kause J, Smith G, Prytherch D, Parr M, Flabouris A, Hillman K, et al. A comparison of antecedents to cardiac arrests, deaths and emergency intensive care admissions in Australia and New Zealand, and the United Kingdom-the ACADEMIA study. Resuscitation 2004;62:275-82.

Meert KL, Donaldson A, Nadkarni V, Tieves KS, Schleien CL, Brilli RJ, et al. Multicenter cohort study of in-hospital pediatric cardiac arrest. Pediatric Critical Care Medicine 2009;10:544-53.

NHSLA. Risk management standards for NHS Trusts providing acute, community or mental health and learning disability services \& non NHS providers of care. Standard 4.8; 2012-2013. p. 111.

NPSA. A review of patient safety for children and young people in hospital. London: National Patient Safety Agency (NPSA), National Reporting and Learning Service; 2009.

Odetola FO, Clark SJ, Dechert RE, Shanley TP. Going back for more: an evaluation of clinical outcomes and characteristics of readmissions to a pediatric intensive care unit. Pediatric Critical Care Medicine 2007;8(4):343-7.

PICANET report 2012 (data from 2011). Paediatric Intensive Care Audit Network. http://www. picanet.org.uk/Documents/ General/Annual_Report_2012/Ninth_PICANet_Annual_

Report_2009_2011_Tables_And_Figures.pdf [accessed 28.08.13].

Roland D, Oliver A, Edwards ED, Mason BW, Powell CVE. Use of paediatric early warning systems in Great Britain: has there been a change of practice in the last 7 years? Archives of Disease in Childhood 2013(August)., http://dx.doi.org/10.1136/archdischild-2012-302783.

Sharek PJ, Parast LM, Leong K, Coombs J, Earnest K, Sullivan J, et al. Effect of a rapid response team on hospital-wide mortality and code rates outside the ICU in a children's hospital. Journal of the American Medical Association 2007;298(19):2267-74.

Slater A, Shann F, Pearson G, for the PIM Study Group. PIM2: a revised version of the Paediatric Index of Mortality. Intensive Care Medicine 2003;29:278-85.

SPSS Inc. . PASW statistics for Windows, version 18.0. Released 2009. Chicago: SPSS Inc; 2009.

Tasker RC. Paediatric cardiac resuscitation: can we do better? Archives of Disease in Childhood 2005;90(11):1102-3.

Tibballs J, Kinney S, Duke T, Oakley E, Hennessy MI. Reduction of paediatric in-patient cardiac arrest and death with a medical emergency team: preliminary results. Archives of Disease in Childhood 2005;90:1148-52.

Tibballs J, Kinney S. Reduction of hospital mortality and preventable cardiac arrest and death on introduction of a paediatric medical team. Pediatric Critical Care Medicine 2009;10:306-12.

Tume L. A 3 year review of emergency PICU admissions from the ward in a specialist cardiorespiratory centre. Care of the Critically III 2004;21(1):4-7.

Tume L. The deterioration of children in ward areas in specialist children's hospital. Nursing in Critical Care 2006;12(1):12-7. 